\title{
New recommendations for supporting old responsibility and transparency values in medical journal publications
}

At the end of August 2013, the International Committee of Medical Journal Editors (ICMJE) published new recommendations for the conduct, reporting, editing, and publication of scholarly work in medical journals. ${ }^{1}$

The ICMJE issued guidelines for the standardization of scientific manuscript format for the first time in 1978. Such guidelines were formerly known as the Uniform Requirements for Manuscripts Submitted to Biomedical Journals. Since its first version, multiple updates have been published. This time, the guideline title was also modified so as to fully express its content and purpose: to review best practices in the conduct and reporting of research studies and to help those involved in the process for distributing accurate, clear and unbiased information.

The most important changes include the addition of a fourth criterion for authorship and urging the scientific community to report studies with negative findings.

As per the Uniform Requirements, all the following conditions had to be met to be considered an author:

- Having made substantial contributions to the conception and design of the work; or the acquisition, analysis, or interpretation of data for the work.

- Having drafted the work or revised it critically for important intellectual content.

- Having approved the final version of the manuscript to be published.

The new criterion implies agreement to be accountable for all aspects of the work related to the accuracy or integrity of the submitted information. This is not just one more requirement; it implies not only taking into consideration individual work or any merit that may be awarded to the publication, it involves being responsible as a team towards the community, professional honesty and considering any consequences that the published information may have on individuals' health. This is consistent with the Declaration of Helsinki, which establishes that authors are ethically obliged to make human research findings publicly available and to be accountable for their reports' integrity and accuracy. ${ }^{2}$

The new recommendations promote that protocols and analysis plan be reviewed before the study publication and that readers have access to such documents once the study is published. This allows to assess reporting accuracy and the validity of results.

Another significant amendment to the guideline is the emphasis on the need to publish studies with negative findings, primarily focused on reporting of results above any interest that may be posed for readers by positive findings. This also reveals the concept of considering results a "public asset," a responsibility of investigators and editors before the general community. Reporting such results also provides "answers" to the community's questions, even if they are not as "expected."

The recommendations refer authors, reviewers and editors to the guidelines for scientific work submission. ${ }^{3}$ Such guidelines, which come together under the international initiative called the Equator Network (Enhancing the Quality and Transparency of Health Research), ${ }^{4}$ make up a valuable resource for reporting different research studies in a complete and accurate manner. This also contributes to transparent research reporting and allows readers to have an active role to judge result validity and scope by themselves.

The lack of research trials and those studies that are inadequately reported or conducted result in a harm for the community, both participating patients and those who may potentially benefit from the results. This is particularly significant in developing regions, where the conduct of research studies is hurdled by scarce resources or by the fact that medical activities are overtaken by the urgency of providing medical care. At the same time, the paradox is that such developing countries are probably the ones where more research "questions" relevant for public health require an urgent answer. One of the medical fields especially affected by the lack of research is pediatrics. ${ }^{5}$ Childhood accounts for a particularly vulnerable period and supposes ethical and operational challenges. It is not a coincidence that an international initiative has been established to provide pediatric research guidelines (StaR Child Health) that allow to improve such situation. ${ }^{6}$ For this reason, any guideline that allows to improve research conduct and reporting of outcome is especially important for those who devote their work to children's health. 
Research is inherent to an unavoidable social responsibility. Outcome reporting and publication, whether positive or negative, are the utmost purpose of research. Contributions made by every work group and study are a valuable element for a specific field of knowledge. However, it is essential to take into account that results are always limited and are part of a bigger body of knowledge. It is necessary to join our efforts and collaborate so as to obtain valid results that can be made available to the rest of the community. Knowledge cannot be built through individual activities but through collective tasks of researchers, authors, reviewers and editors, all of which imply obligations to the society. Any initiative that promotes that each party takes responsibility for the work, the transparency that allows others to critically appraise results, and the possibility of publishing studies with negative findings, implies an action towards having common evidence available for the benefit of all patients.

María Elina Serra, M.D. Assistant Editor
1. Recommendations for the Conduct, Reporting, Editing, and Publication of Scholarly Work in Medical Journals (ICMJE recommendations) [Accessed on: september 19, 2013]. Available at:http://www.icmje.org/urm_main. html.

2. Asociación Médica Mundial. Declaración de Helsinki. Principios éticos para la Investigación Médica en Seres Humanos. Seúl: 59a Asamblea General; octubre 2008. [Accessed on: september 19, 2013]. Available at: http:/ / www.wma. net/es/30publications/10policies/b3.

3. Simera I, Altman, D. G. Reporting medical research. Int J ClinPract, aug. 2013; 67(8):710-716.

4. EQUATOR Network. [Accessed on: september 14, 2013]. Available at:http:/ /www.espanol.equator-network.org/.

5. Martinez-Castaldi C., Silverstein M, Bauchner H. Child versus adult research: the gap in high-quality study design. Pediatrics 2008;122;52-7.

6. Hartling L, Wittmeier K, Caldwell P., Van der Lee H,et al. star child health: developing evidence-based guidance for the design, conduct, and reporting of pediatric trials. $P e-$ diatrics 2012;129;S112. 